\title{
Optimierung von Aufnahmeparametern mittels projektionsbasierter Qualitätskenngrößen in der industriellen Computertomographie
}

\author{
Alexandra Krämer ${ }^{1}$, Pascal Böhmler $^{1}$, Gisela Lanza ${ }^{1}$ \\ ${ }^{1}$ wbk Institut für Produktionstechnik, Karlsruher Institut für Technologie (KIT), Karlsruhe, Deutschland
}

\begin{abstract}
Zusammenfassung
Die Röntgen-Computertomographie gewinnt in der industriellen Messtechnik immer mehr an Bedeutung. Allerdings sind das Messergebnis und die dazugehörige Messabweichung stark von der Wahl der Aufnahmeparameter abhängig, die durch den Anwender getroffen wird. Daher wird eine Methodik vorgestellt, die eine automatisierte Einstellung der Aufnahmeparameter mit dem Ziel einer jeweils verringerten Messabweichung ermöglicht. Mithilfe von Bildqualitätsparametern kann die Bildqualität von Einzelprojektionen bewertet und diese im Rahmen eines experimentellen Modells mit der merkmalspezifischen Messabweichung der Einzelmessung am rekonstruierten 3D-Volumen verknüpft werden. Aus dem Modell kann dann die optimale Bildqualität der Einzelprojektionen abgeleitet werden, auf deren Basis dann automatisiert und messaufgabenspezifisch ein bauteil- und geräteabhängiges Set an optimalen Aufnahmeparametern bestimmt wird. Die hierfür notwendige Beurteilung der Bildqualität von Einzelprojektionen kann nur mit objektiven Kennwerten erfolgen. Im Beitrag werden daher verschiedene Kenngrößen zur Bewertung der Bildqualität vorgestellt und auf ihre Anwendbarkeit in der Computertomographie überprüft.
\end{abstract}

Keywords: Computertomographie, Messabweichung, Aufnahmeparameter, Bildqualität

\section{Einleitung}

Immer komplexere Fertigungsprozesse sowie der Einsatz neuer Technologien (beispielsweise additive Fertigungsverfahren) ermöglichen die Herstellung von Bauteilen mit verstärkter Funktionsintegration. Diese weisen oft innere Strukturen und verdeckte Merkmale auf, die für konventionelle taktile Messtechnik nur schwer bzw. nicht mehr zugänglich sind. Der Einsatz der Röntgen-Computertomographie (CT) in der dimensionellen Messtechnik rückt daher in den Vordergrund, da dieses Verfahren die Möglichkeit zu einer ganzheitlichen und vollständigen Datenerfassung innerer und äußerer Bauteilmerkmale bietet [1].

Computertomographische Messverfahren ermöglichen im Vergleich zu taktiler Messtechnik eine deutlich höhere Datendichte, da mit innen eine große Anzahl an Datenpunkten auf der gesamten Oberfläche aufgenommen werden kann und somit ein ganzheitliches, volumetrisches 3D-Modell des Bauteils zur Verfügung steht.

Die Röntgen-Computertomographie ist dabei ein indirektes Messverfahren, dessen Messergebnis durch eine Vielzahl von Faktoren beeinflusst wird. Die vom Anwender beeinflussbaren Aufnahmeparameter, beispielsweise Bauteilorientierung und -position sowie Röhrenstrom, Röhrenspannung oder Belichtungsdauer, stehen zueinander in Wechselwirkung und haben einen signifikanten Einfluss auf das Messergebnis und die dazugehörige Messabweichung [1-5].

In der industriellen Praxis ist die Wahl geeigneter Aufnahmeparameter dabei abhängig vom persönlichen Erfahrungswissen des Anwenders und stellt daher trotz zunehmender Praxiserfahrung weiterhin eine große Herausforderung dar [6]. Aufgrund des komplexen Messverfahrens kann der Einfluss der Parameterwahl nicht direkt bestimmt werden. Dadurch können mehrere Messungen desselben Bauteils zu deutlich unterschiedlichen Ergebnissen führen. Eine Bewertung der Parameterwahl ist momentan allerdings erst nach der Messung und Rekonstruktion möglich. Fällt die Bewertung negativ aus, muss die Messung ggf. zeitaufwändig wiederholt werden. In diesem Beitrag wird daher eine Methodik zur automatisierten Einstellung der Aufnahmeparameter in der industriellen Computertomographie mit dem Ziel einer verringerten Messabweichung vorgestellt. Dies soll bauteilspezifisch bereits vor der eigentlichen 
Messung, auf Basis von wenigen Einzelprojektionen, geschehen. Dazu werden die Einzelprojektionen bezüglich ihrer Bildqualität bewertet.

Der Beitrag ist wie folgt gegliedert: Zunächst wird ein Überblick über den projektionsbasierten Ansatz zur Optimierung von Aufnahmeparametern gegeben (Abschnitt 2). In Abschnitt 3 wird der Einfluss der Bildqualität in der Computertomographie umrissen, bevor in Abschnitt 4 verschiedene Bildqualitätskenngrößen eingeführt und in Abschnitt 5 experimentell auf ihre Anwendbarkeit in der Computertomographie getestet werden. Die Resultate werden in Abschnitt 6 vorgestellt und in Abschnitt 7 diskutiert.

\section{Ansatz zur Optimierung von Aufnahmeparametern}

Mithilfe von Bildqualitätskenngrößen kann die Bildqualität von Einzelprojektionen bewertet und diese in einem experimentellen Modell mit der merkmalspezifischen Messabweichung der Einzelmessung am rekonstruierten 3DVolumen verknüpft werden.

Zunächst müssen daher geeignete Bildqualitätskenngrößen ausgewählt und auf ihre Eignung zur Bewertung der computertomographischen Messung überprüft werden. Dies wird in diesem Beitrag näher erläutert.

Nach der Auswahl geeigneter Bildqualitätskenngrößen wird der Zusammenhang zwischen der Bildqualität der Projektionen und der merkmalspezifischen Messabweichung am rekonstruierten 3D-Volumen erforscht. Die für die experimentelle Modellbildung notwendigen Versuchsdaten werden im Rahmen systematischer Messungen anhand von verschiedenen bauteilähnlichen Probekörpern aufgenommen (vgl. [7]). Ein Regressionsmodell wird zur Modellierung der Zusammenhänge verwendet und durch Varianz- und Sensitivitätsanalyse überprüft.

Aus dem Modell soll bezogen auf Maß- und Formmessung die zu erstrebende Bildqualität der Einzelprojektionen abgeleitet werden, auf deren Basis dann messaufgabenspezifisch ein bauteil- und geräteabhängiges Set an optimalen Aufnahmeparametern bestimmt wird. Daher wird ein allgemeingültiges Optimierungsproblem für Aufnahmeparameter am Computertomographen abgeleitet, das eine möglichst geringe Messabweichung für die jeweilige Messaufgabe liefert.

Hierzu werden aus dem entwickelten Modell die Werte der Bildqualitätsparameter so ausgewählt, dass sie die jeweils geringste
Messabweichung zur Folge haben. Daraufhin erfolgen eine Normierung der Qualitätskenngrößen und die Festlegung eines Grenzbereichs, der die jeweils noch akzeptablen Werte der Bildqualitätskenngrößen beschreibt. Der Grenzbereich kann beispielsweise aus einer Sensitivitätsanalyse abgeleitet werden.

Die normierten Qualitätskenngrößen werden nun in einer gewichteten Summe zu einer übergeordneten Zielgröße für die Optimierung zusammengefasst. Unterschiedliche hinterlegte Funktionen zur Gewichtung der Zielgrößen können die Bedeutung der einzelnen Bildqualitätskenngrößen für das Messergebnis abbilden.

Die in der Optimierung betrachteten Aufnahmeparameter sind hierbei Röhrenstrom, Röhrenspannung, Belichtungsdauer, Verstärkung, Auflösung und Position des Bauteils.

Die Einstellung der Aufnahmeparameter erfolgt dabei anhand der als besonders kritisch identifizierten Projektionen (beispielsweise die Projektionen mit größter und kleinster Durchstrahlungslänge). Die gesamtoptimale Einstellung der Parameter ist dabei immer bauteilund messaufgabenspezifisch und jeweils ein Kompromiss zwischen den optimalen Einstellungen der kritischen Einzelprojektionen.

Zusätzlich muss der Lösungsraum auf den realen Raum von Möglichkeiten eingeschränkt werden. Dies erfolgt durch die Berücksichtigung von gerätespezifischen Restriktionen wie beispielsweise Messbereich und Verfahrwege sowie Minimal- und Maximalwerte von Röhrenspannung und -strom. Die Lösung erfolgt mittels eines genetischen Algorithmus.

\section{Bildqualität in der Computertomographie}

Kontrast, Rauschen und Bildschärfe sind wichtige Merkmale zur Beschreibung der Bildqualität von Einzelprojektionen und des rekonstruierten Volumens. Alle Parameter im tomographischen Abbildungsprozess (z.B. Hardware und Aufnahmeparameter) beeinflussen die jeweilige Bildqualität der Projektionen und haben somit einen großen Einfluss auf das computertomographische Messergebnis [8]. Betrachtet man das 3DVolumen, so kommen noch Rekonstruktionseinflüsse (beispielsweise Artefakte) hinzu, die ebenfalls Auswirkungen auf die Bildqualität des 3D-Volumens haben [9]. Simulative Untersuchungen von Hiller et al. [10] zum Einfluss der Bildqualität auf die Messabweichung zeigen beispielsweise, dass mit zunehmender Detektorunschärfe die Messabweichung für den Durchmesser einer Kugel zunimmt. 
Im Nachfolgenden werden einige Ansätze zur Beschreibung der Bildqualität durch objektive Qualitätskenngrößen beschrieben. Für die industrielle Computertomographie schlägt die DIN EN 16016 ausgehend von der Bildqualität der Einzelprojektion eine transmissionsbasierte Parametereinstellung vor [11]. Ein optimales Signal-Rausch-Verhältnis soll durch eine minimale Transmission von $10 \%$ bis $20 \%$ in der Einzelprojektion erreicht werden. Arbeiten von Reiter et al. [12] zeigen allerdings, dass unterschiedliche Einstellungen der Aufnahmeparameter (z.B. Röhrenspannung und Belichtungsdauer) bei gleicher Transmission zu sehr unterschiedlichem Signal-RauschVerhältnis sowie zu unterschiedlicher Bildqualität führen können. Die Transmission ist daher als alleiniger Parameter zur Beschreibung der Bildqualität nicht geeignet.

Für eine Evaluation des rekonstruierten Volumens existieren allerdings bereits verschiedene Histogramm-basierte [13-15] und kantenbasierte Kennwerte [16, 17].

Zusammenfassend kann festgehalten werden, dass die Qualität der Einzelprojektionen einen direkten Einfluss auf die Qualität des rekonstruierten 3D-Volumenmodells hat. Bildmerkmale wie Rauschen, Kontrast und Bildschärfe können dabei durch Kenngrößen beschrieben und zur Bewertung der Einzelprojektionen sowie des rekonstruierten Volumens herangezogen werden.

\section{Beurteilung der Projektionsqualität}

Wie bereits erwähnt, reicht die Betrachtung von einzelnen Qualitätskenngrößen für eine objektive und eindeutige Beschreibung der Bildqualität nicht aus (vgl. [18]). Daher müssen geeignete objektive, referenzfreie Bildqualitätskenngrößen identifiziert, daraus geeignete Größen ausgewählt und als kombiniertes Maß für die Bildqualität verwendet werden. Neben Kontrast und Rauschen spielt die Bildschärfe ebenfalls eine große Rolle [9, 10]. Hierbei können sowohl lokale als auch globale Größen zur Bewertung herangezogen werden.

Für Multimedia-Anwendungen kann auf vergleichende Untersuchungen von Verfahren zur Bildbewertung (Image Quality Assessment IQA) (vgl. [19-21]) und IQA-Datenbanken mit subjektiven Referenzbewertungen (z.B. [22]) zurückgegriffen werden. Eine subjektive visuelle Bewertung der Einzelprojektionen in der industriellen CT durch den Anwender kann im Gegensatz dazu eine Falscheinschätzung der tatsächlichen Bildqualität hervorrufen [11, 23].
Im Folgenden werden daher ausgewählte Kenngrößen zur Beschreibung der Bildschärfe vorgestellt, die auf ihre Eignung zur Bewertung von computertomographischen Projektionen geprüft werden:

Der globale Kennwert der Entropie beschreibt den Informationsgehalt eines Signals. Ist $p_{i}$ die Häufigkeit eines Grauwertes $i$ der computertomographischen Projektion, dann ist die Entropie $H$ [24]:

$$
H=-\sum_{i} p_{i} \log _{2}\left(p_{i}\right)
$$

Die Entropie ist dabei ein Maß für die Verteilung der Grauwerte in der Einzelprojektion. Eine niedrige Entropie weist auf einen hohen Kontrast bzw. eine geringe Streuung der Grauwerte hin (vgl. auch [14]).

Wird das Bild vor der Kennwertberechnung in einzelne Segmente (Blöcke) zerlegt, spricht man von Verfahren in segmentierten Bildbereichen. Diese Verfahren beurteilen die lokale Projektionsqualität in Segmenten gleicher Größe. Eine beschränkte Ortsinformation bleibt also erhalten.

Neben einer lokalen Qualitätsaussage kann durch Kombination der lokalen Information auch ein globaler Kennwert für die Projektion bestimmt werden. Die prinzipielle Vorgehensweise besteht aus Blockbildung, Blockauswahl, lokaler Berechnung und Kombination zu einem globalen Kennwert. Es ist möglich, das gesamte Bild in die Berechnung miteinzubeziehen oder bestimmte Segmente für die Auswertung auszuwählen. Um die Bildschärfe zu bewerten, werden nur die Segmente, die Objektkanten beinhalten, ausgewertet, wie bei der Ermittlung der lokalen Varianz der Grauwerte (LVAR). Grundannahme bei der Verwendung der Varianz als Schärfekennwert ist, dass ein Segment mit scharfer Kante große Unterschiede in den Intensitätswerten aufweist. Mit zunehmender Unschärfe werden Kanten und Übergänge zwischen Intensitätswerten geglättet und die Varianz im Segment nimmt ab. Der größte Wert der Varianz wird daher für ein scharfes Bild angenommen [19, 25]. Die lokale Varianz $L V A R_{\text {seg }}$ eines diskreten Bildsegments $g_{s}(x, y)$ berechnet sich nach:

$$
L V A R_{\text {seg }}=\frac{1}{i j} \sum_{x=1}^{i} \sum_{y=1}^{j}\left(g_{s}(x, y)-\mu\right)^{2}
$$

wobei $\mu$ den mittleren Grauwert im Segment darstellt. Als Segmentgröße $i$ x $j$ werden $16 \times 16$ Pixel gewählt. 
Zur Bestimmung eines globalen Kennwertes LVAR wird das Ergebnis aller Segmente, die eine erkannte Objektkante beinhalten, gemittelt. Eine weitere große Klasse der Kennwerte zur Beurteilung der Bildschärfe bilden differenzenbasierte Verfahren. Diese Verfahren lassen sich einfach implementieren und finden sich häufig in der Literatur [25].

Für die Beurteilung der Schärfe von Bildern werden sowohl Differenzen erster Ordnung (gradientenbasierte Verfahren) als auch zweiter Ordnung eingesetzt. Differenzenbasierte Kennwerte entstehen durch die Summierung der Differenzen im Bild. Mit zunehmender Unschärfe werden Intensitätswerte benachbarter Pixel gemittelt. Die Differenz zwischen hellen und dunklen Objekten nimmt ab [26]. Ein höherer Kennwert wird daher mit einem schärferen Bild assoziiert [19].

Der normalisierte, quadrierte Gradientenbetrag (squared gradient SG) einer Projektion $g(x, y)$ der Größe M x N Pixel ist:

$$
S G=\frac{1}{M N} \sum G(x, y)^{2}
$$

Der Gradientenbetrag $G(x, y)$ wird durch den Sobel-Operator approximiert. GI. (3) entspricht damit der Tenengrad-Funktion [27].

Die Sum of Modified Laplacian (SML) basiert auf Differenzen zweiter Ordnung und besitzt ein stark ausgeprägtes Maximum für scharfe Bilder $[18,28,29]$ :

$$
\begin{aligned}
& S M L= \\
& \sum(|-g(x+1, y)+2 g(x, y)-g(x-1, y)| \\
& +|-g(x, y+1)+2 g(x, y)-g(x, y-1)|)
\end{aligned}
$$

Auch eine direkte Bewertung der Kante ist möglich. Es existieren daher Kennwerte zur Beurteilung der Bildschärfe, die die Kantenregion eines Bildes analysieren [30]. Die durchschnittliche Kantenstärke (DKS) berechnet sich aus dem Verhältnis der Summe der Kantenbreite $b_{i}$ aller Kantenpixel und der Anzahl $K$ der Kantenpixel:

$$
D K S=\frac{1}{K} \sum_{i=1}^{K} b_{i}
$$

Die Kantenbreite $b_{i}$ ist die Pixelanzahl zwischen lokalen Extrema ausgehend vom Kantenpixel in Gradientenrichtung [31].

Unschärfe beeinflusst außerdem die Korrelation benachbarter Pixel. Mit der Autokorrelationsfunktion $a(i, j)$ der Projektion $g(x, y)$ kann diese daher durch den Autokorrelationskennwert $A K K$

$$
\begin{aligned}
& a_{\min }=\min (a(i, j)) ; \quad i, j \in[-n, n] \\
& A K K=\frac{1}{4 n^{2}} \sum_{\substack{i=-n \\
i \neq 0}}^{n} \sum_{\substack{j=-n \\
j \neq 0}}^{n}\left(a(i, j)-a_{\min }\right)
\end{aligned}
$$

bewertet werden [25]. Der Parameter $n$ wird in Vorversuchen empirisch bestimmt und auf $n=$ 16 festgelegt.

\section{Experimenteller Aufbau}

Als Prüfkörper wird ein messtechnisch gut beherrschbares Objekt mit hochpräzisen Rubinkugeln verwendet. Dieses hat den Vorteil, dass bei korrekter Ausrichtung entlang der Rotationsachse des Drehtisches im Computertomographen alle Winkelstellungen ähnliche Einzelprojektionen erzeugen. Dies ermöglicht es, zunächst eine prinzipielle Aussage über den Einfluss der Bildqualität auf die Messabweichung zu treffen. Der Prüfkörper besteht aus einer Anordnung von drei Rubinkugeln, die auf Kohlefaser-Schäfte geklebt sind. Messmerkmal ist der Durchmesser der mittleren Kugel (Abb.1(a)), der zuvor mittels taktiler Messung bestimmt wurde. Die taktile Referenzmessung ergibt einen Durchmesser der Rubinkugel von 1,9920 mm gemittelt über 25 Wiederholmessungen mit einer Standardunsicherheit von $1,2910 \times 10^{-5}$ $\mathrm{mm}$. Die anderen beiden Kugeln dienen der Objekt-Ausrichtung.

Für den Prüfkörper werden 20 CT-Messungen mit einem Zeiss Metrotom 800 mit Wolframanode durchgeführt. Die Pixelgröße des Detektors ist $127 \times 127 \mu \mathrm{m}$ bei einer Auflösung von $1536 \times 1920$ Pixel.

Es wird ein randomisiertes Face Centered Central Composite Design (FCCCD) zur Variation der Aufnahmeparameter verwendet. Röhrenspannung $U$, Röhrenstrom $I$ und Vergrößerung $V g$ werden gemäß des Versuchsplans verändert (siehe Tab. 1). Konstante Parameter sind die Integrationszeit $T=1000 \mathrm{~ms}$, die Anzahl der Projektionen pro Messung $N=1550$ und die DetektorVerstärkung (Gain) von 2,5. Binning und Bildmittelung werden nicht genutzt. 
Tab. 1: FCCCD-Versuchsplan

\begin{tabular}{rrrr}
\hline Nr. & $\mathrm{U}[\mathrm{kV}]$ & $\mathrm{I}[\mu \mathrm{A}]$ & $\mathrm{Vg}$ \\
\hline 1 & 80 & 60 & 12,46 \\
2 & 130 & 60 & 12,46 \\
3 & 80 & 110 & 12,46 \\
4 & 130 & 110 & 12,46 \\
5 & 80 & 60 & 14,23 \\
6 & 130 & 60 & 14,23 \\
7 & 80 & 110 & 14,23 \\
8 & 130 & 110 & 14,23 \\
9 & 80 & 85 & 13,345 \\
10 & 130 & 85 & 13,345 \\
11 & 105 & 60 & 13,345 \\
12 & 105 & 110 & 13,345 \\
13 & 105 & 85 & 12,46 \\
14 & 105 & 85 & 14,23 \\
15 & 105 & 85 & 13,345 \\
16 & 105 & 85 & 13,345 \\
17 & 105 & 85 & 13,345 \\
18 & 105 & 85 & 13,345 \\
19 & 105 & 85 & 13,345 \\
20 & 105 & 85 & 13,345
\end{tabular}

Um das Verhalten der Kennwerte unter definierten Rahmenbedingungen untersuchen zu können, wird die Qualität der Projektionen künstlich verändert. Hierzu wird eine künstliche Unschärfe der Projektionen simuliert. Auf computertomographische Projektionen wird dazu ein Gauß-Filter mit variabler Filtergröße $F S$ und Standardabweichung $\sigma$ angewendet. $\sigma$ wird zwischen $\sigma_{1}=0,1$ und $\sigma_{100}=10$ in Schritten von $\Delta \sigma=0,1$ variiert. Die Filtergröße $F S$ berechnet sich aus

$$
F S=2\lceil 2 \sigma\rceil+1
$$

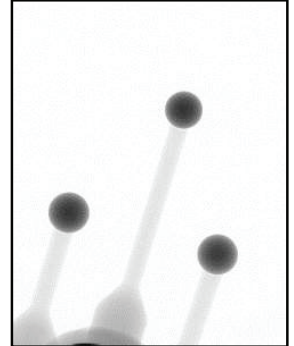

(a)

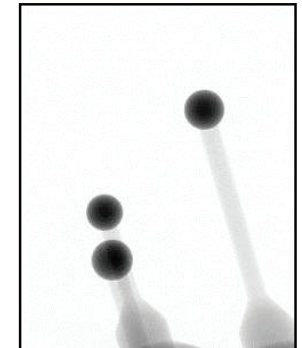

(b)
Abb. 1: Projektion 1 (a) und Projektion 2 (b) des Prüfkörpers

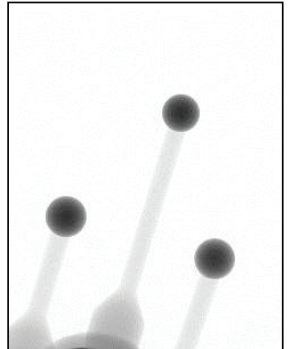

(a)

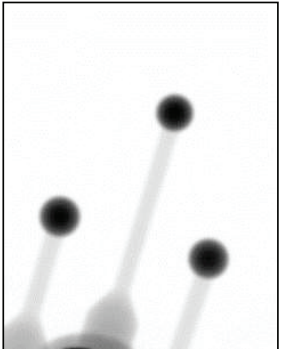

(b)
Abb. 2: Projektion 1 aus Abb.1(a) nach Anwenden des Gauß-Filters. Filterparameter: (a) $\sigma=1, F S=5$ und (b) $\sigma=10$, $F S=41$

Es werden zwei Projektionen der Messung 19 (Zentralpunkt des FCCCD-Versuchsplans) gewählt. Zwischen den Projektionen ist der Prüfkörper um $90^{\circ}$ gedreht (Abb. 1).

Für geeignete Kennwerte wird erwartet, dass sie bei Anwendung eines Gauß-Filters mit monoton zunehmender Standardabweichung $\sigma$ ebenfalls monotones Verhalten zeigen.

In einer zweiten Untersuchung wird die Korrelation der Kennwerte der 20 Messungen mit der Messabweichung des Kugeldurchmessers überprüft. Als Messabweichung wird die Differenz des CT-Messwertes zu dem taktil bestimmten gemittelten Referenzwert verwendet.

Für jede Projektion einer Messung werden die Kennwerte einzeln berechnet und dann über alle Projektionen gemittelt.

Die Auswertung des rekonstruierten 3DVolumens erfolgt mit einer lokal-adaptiven Schwellwertbestimmung, wobei ein zuvor bestimmter globaler ISO50-Schwellwert als Initialwert dient und dieser in einem gewählten Suchabstand von zwei Voxeln korrigiert wird. Die Durchmesserbestimmung der Kugel erfolgt mittels Gauß-Einpassung.

\section{Ergebnisse}

Die Auswertung der simulierten Unschärfe zeigt, dass mit Ausnahme der Entropie und der lokalen Varianz die untersuchten Kennwerte dazu geeignet sind, die Bildschärfe über einen großen Schärfebereich zu beurteilen.

Die differenzenbasierten Kennwerte SG und $S M L$ zeigen bereits bei geringer Unschärfe ein gutes Ansprechverhalten (Abb. 3 (c-d)). Die Entropie einer Projektion nimmt bis zu einer Grenz-Standardabweichung $(\sigma \approx 0,7)$ monoton zu und nach Annahme des Maximums monoton ab (Abb.3(a)). Dies liegt darin begründet, dass mit zunehmender Unschärfe zunächst die Grauwerte im Bereich der Objektkante in den Hintergrund gestreut werden und die Entropie 
entsprechend zunimmt. Mit weiter steigender Unschärfe nehmen benachbarte Pixel zunehmend gleiche Grauwerte an und die Entropie sinkt entsprechend. Die DKS verhält sich daher gegenläufig zu den anderen Kennwerten und nimmt für die schärfste Projektion ein Minimum an (Abb. 3(e)).

Die lokale Varianz LVAR ist von Streuungen überlagert (Abb.3 (b)). Dies ist durch die Wahl der Segmente zu begründen. Insbesondere für große Unschärfe werden nicht alle Objektkanten korrekt erkannt, d.h. die Zahl der beurteilten Segmente ändert sich mit zunehmender Unschärfe.

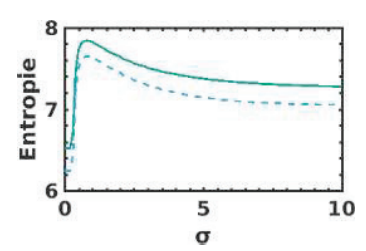

(a)

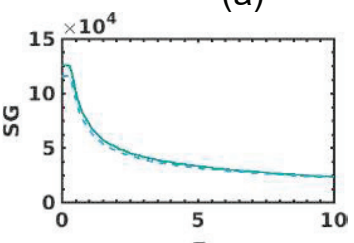

(c)

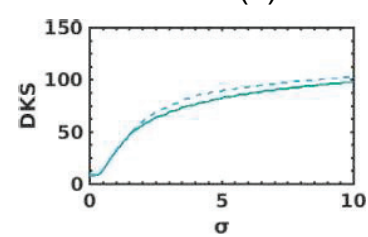

(e)

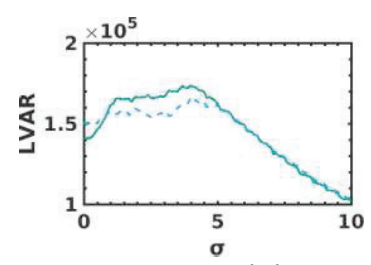

(b)

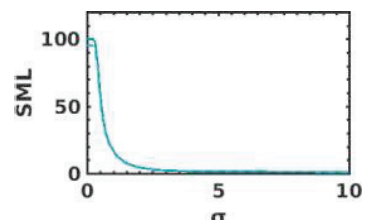

(d)

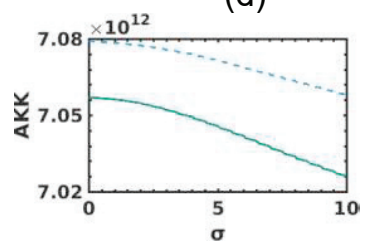

(f)
Abb. 3: Kennwerte für simulierte Unschärfe: (a) Entropie; (b) LVAR; (c) SG; (d) SML; (e) DKS; (f) AKK. Projektion 1: Volllinie, Projektion 2 : Strichlinie.

Für den Prüfkörper weisen $L V A R$, SG und $D K S$ eine statistisch signifikante monotone Korrelation (Signifikanzniveau $\alpha=0,05$ ) mit der Messabweichung auf (Abb. 4 / Tab. 2). Die Korrelation ist für die LVAR am stärksten ausgeprägt. Als besonders vielversprechend für den Einsatz als Optimierungskriterium der Scan-Parameter für einfache WerkstückGeometrien können somit die lokale Varianz LVAR und der squared gradient SG identifiziert werden. Die Mittelung der Kennwerte über die Projektionen einer Messung stellt ein vereinfachtes Vorgehen dar. Für das untersuchte Prüfobjekt ist diese Vereinfachung zulässig, da die Streuung der Kennwerte gering ist. Der Variationskoeffizient für alle Kennwerte ist kleiner 0,1.

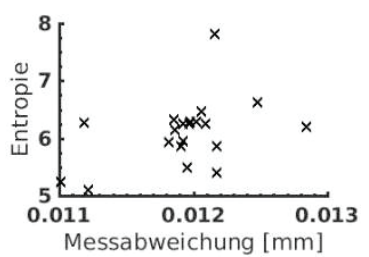

(a)

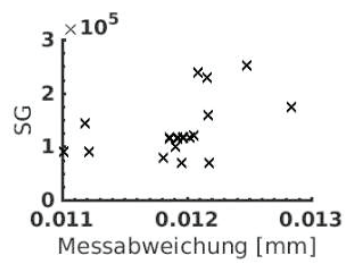

(c)

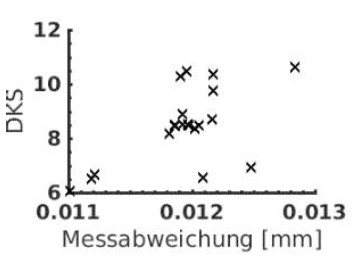

(e)

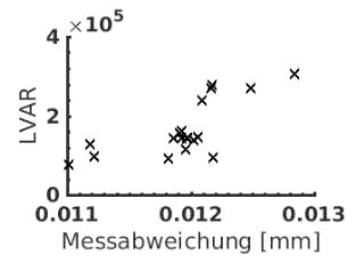

(b)

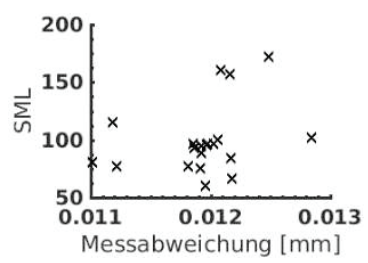

(d)

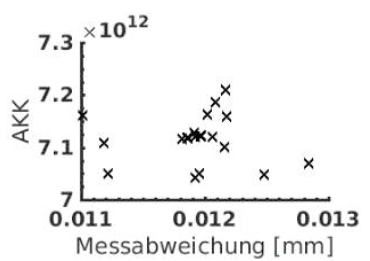

(f)
Abb. 4: Kennwerte und Messabweichung für Prüfkörper 1 [(a) Entropie; (b) LVAR; (c) SG; (d) SML; (e) DKS; (f) AKK]

Tab. 2: Rangkorrelation nach Kendall ( $\left.T_{b}\right)$ der Kennwerte mit der Messabweichung

\begin{tabular}{lcc}
\hline & $\boldsymbol{T}_{\mathbf{b}}$ & $\boldsymbol{p}$-Wert \\
\hline Entropie & 0,2797 & 0,0914 \\
LVAR & 0,5646 & 0,0006 \\
SG & 0,4169 & 0,0113 \\
SML & 0,2797 & 0,0914 \\
DKS & 0,3536 & 0,0322 \\
AKK & 0,1270 & 0,4551
\end{tabular}

\section{Zusammenfassung und Ausblick}

Es wurde gezeigt, dass sich Kennwerte zur Bewertung der Bildschärfe auf CTAnwendungen übertragen lassen und in der Lage sind, Unschärfe korrekt zu bewerten. Manche Kennwerte sind zudem zur Messabweichung korreliert.

Anzumerken ist, dass diese Ergebnisse sich ohne weitere Untersuchungen nicht auf beliebige Werkstück-Geometrien übertragen lassen. Während die Streuung der vorgestellten Kennwerte für den geometrisch einfachen Prüfkörper Rubinkugel gering ist, wird an 
Untersuchungen mit komplexeren Prüfgeometrien insbesondere für Kennwerte mit Kantenerkennung ( $L V A R, D K S)$ eine deutliche größere Streuung festgestellt.

Daher sollten globale Kennwerte bzw. global gemittelte lokale Kennwerte nicht alleinig zur Optimierung der Scan-Parameter genutzt werden.

Eine Mittelung der Kennwerte aller Projektionen ist ebenfalls nur für einfache Geometrien, bei denen die Projektionen eine große Ähnlichkeit aufweisen, zielführend. Durch die Abbildung komplexer Bauteils auf den Detektor entstehen unterschiedliche Projektionen, deren Kennwerte sich ebenso unterscheiden.

In weiteren Untersuchungen soll daher die lokale und projektionsindividuelle Auswertung von Kennwerten, insbesondere für komplexe Geometrien, angestrebt werden.

Durch eine geeignete Wahl und Auswertung von projektionsbasierten Kenngrößen ist es dann möglich, einen Zusammenhang zur Messabweichung von Geometriemerkmalen herzustellen. Dies kann für die projektionsbasierte Optimierung von Aufnahmeparametern verwendet werden, um die Messabweichung bei computertomographischen Messungen $\mathrm{zu}$ verringern und die $\mathrm{Re}-$ produzierbarkeit der Messungen zu erhöhen.

\section{Literaturnachweis}

[1] J.-P Kruth, M. Bartscher, S. Carmignato, R. Schmitt, L. De Chiffre, A. Weckenmann, 'Computed tomography for dimensional metrology', CIRP Annals - Manufacturing Technology, 60 (2), 821-842 (2011); doi:10.1016/j.cirp.2011.05.006

[2] J. Angel, L. De Chiffre, 'Comparison on Computed Tomography using industrial items', CIRP Annals - Manufacturing Technology, 63 (1), 473-476 (2014); doi:10.1016/j.cirp.2014.03.034

[3] L. De Chiffre, S. Carmignato, J.-P. Kruth, R. Schmitt, A. Weckenmann, 'Industrial applications of computed tomography', CIRP Annals Manufacturing Technology, 63 (2), 655-677 (2014); doi:10.1016/j.cirp.2014.05.011

[4] R. Schmitt, C. Niggemann, 'Uncertainty in measurement for $\mathrm{x}$-ray-computed tomography using calibrated work pieces', Measurement Science and Technology, 21 (5), 1-9 (2010); doi:10.1088/0957-0233/21/5/054008

[5] A. Weckenmann, P. Krämer, 'Assessment of measurement uncertainty caused in the preparation of measurements using computed tomography', Proceedings of the XIX IMEKO World Congress 'Fundamental and Applied Metrology', Lissabon, 1888-1892 (2009)

[6] VDI/VDE-Gesellschaft Mess- und Automatisierungstechnik (GMA), Fertigungsmesstechnik 2020, TechnologieRoadmap für die Messtechnik in der industriellen Produktion, VDI-Verlag GmbH, Düsseldorf (2011); ISBN 978-3-00-034706-1

[7] A. Krämer, A. Batra, P. Griesz, G. Lanza, 'Development of test bodies for deployment in industrial Computed Tomography'. Conference on Industrial Computed Tomography (iCT) 2016, Wels: Proceedings (2016)

[8] J. Hiller, P. Hornberger, 'Measurement accuracy in X-ray computed tomography metrology: Towards a systematic analysis of interference effects in tomographic imaging', Precision Engineering (2016); doi:10.1016/j.precisioneng.2015.12.003

[9] Y. Tan, K. Kiekens, F. Welkenhuyzen, J. Angel, L. De Chiffre, J.-P. Kruth, W. Dewulf, 'Simulation-aided investigation of beam hardening induced errors in CT dimensional metrology', Measurement Science and Technology, 25(6), 064014 (2014); doi:10.1088/0957-0233/25/6/064014

[10] J. Hiller, T. O. J. Fuchs, S. Kasperl, L. M. Reindl, 'Einfluss der Bildqualität röntgentomographischer Abbildungen auf Koordinatenmessungen: Grundlagen, Messungen und Simulationen', Technisches Messen, 78, 334-347 (2011); doi:10.1524/teme.2011.0137

[11] Deutsches Institut für Normung, Zerstörungsfreie Prüfung - Durchstrahlungsverfahren Computertomographie, DIN EN 16016-1, DIN EN 16016-2, DIN EN 16016-3, DIN EN 16016-4 Beuth Verlag, Berlin (2011)

[12] M. Reiter, M. Krumm, S. Kasperl, C. Kuhn, M. Erler, D. Weiß, C. Heinzl, C. Gusenbauer, J. Kastner, 'Evaluation of transmission based image quality optimisation for X-ray computed tomography', Conference on Industrial Computed Tomography (iCT) 2012, Wels: Proceedings (2012)

[13] M. Reiter, D. Weiß, C. Gusenbauer, M. Erler, C. Kuhn, S. Kasperl, J. Kastner, 'Evaluation of a histogram-based image quality measure for Xray computed tomography,. Conference on Industrial Computed Tomography (iCT) 2014, Wels: Proceedings (2014)

[14] L. Xue, H. Suzuki, Y. Ohtake, H. Fujimoto, M. Abe, O. Sato, T. Takatsuji, 'Quality evaluation of X-ray computed tomography volume data in dimensional metrology'. Conference on Industrial Computed Tomography (iCT) 2016, Wels: Proceedings (2016)

[15] R. Schielein, G. Scholz, C. Kretzer, T. Fuchs, S. Kasperl, F. Bär, S. Kirsch, M. Zepf, M. WoltersRosbach, 'The MUSICES Project: Simulative automated CT acquisition planning for historical brass instruments improves image quality'. Conference on Industrial Computed Tomography (iCT) 2016, Wels: Proceedings (2016)

[16] T. Schönfeld, M. Bartscher, 'Verification and application of quality measures in dimensional $\mathrm{CT}^{\prime}$, International Symposium on Digital Industrial Radiology and Computed Tomography 2015, Dt. Ges. für Zerstörungsfreie Prüfung, Berlin (2015) 
[17] M. Fleßner, A. Müller, E. Hausotte, ‘Evaluating and visualizing of the quality of surface points determined from computed tomography volume data'. MacroScale (2014);

doi:10.7795/810.20150223A

[18] A. Krämer, G. Lanza, 'Projection based evaluation of CT image quality in dimensional metrology', Digital Industrial Radiology and Computed Tomography (DIR 2015) e-Journal of Non-destructive Testing, ISSN 1435-4934 (2015)

[19] R. Ferzli, L. J. Karam, 'A No-Reference Objective Image Sharpness Metric Based on the Notion of Just Noticeable Blur (JNB)', IEEE Transactions on Image Processing 18/4, 717728 (2009); doi: 10.1109/TIP.2008.2011760

[20] S. Yousefi, M. Rahman, N. Kehtarnavaz, 'A new auto-focus sharpness function for digital and smart-phone cameras', IEEE Transactions on Consumer Electronics 57/3, 1003-1009 (2011); doi: 10.1109/TCE.2011.6018848

[21] M. Shahid, A. Rossholm, B. Lövström, H. J. Zepernick, 'No-reference image and video quality assessment: a classification and review of recent approaches', EURASIP Journal on Image and Video Processing, 1-32 (2014); doi: 10.1186/1687-5281-2014-40

[22] H. R. Sheikh, Z. Wang, L. Cormack, A. C. Bovik, Live Image Quality Assessment Database Release 2, (2005); online: http://live.ece.utexas.edu/research/quality

[23] P. Krämer, Simulationsgestützte Abschätzung der Genauigkeit von Messungen mit der Röntgen-Computertomographie. Dissertation, Universität Erlangen-Nürnberg, Erlangen (2012)

[24] T. M. Cover, J. A. Thomas, Elements of Information Theory, 2nd Edition, WileyInterscience, Hoboken, NJ, 13-14 (2006)

[25] C. F. Batten, Autofocusing and astigmatism correction in the scanning electron microscope. Dissertation, Faculty of the Department of Engineering, University of Cambridge (2000)

[26] A. Santos, C. Ortiz De Solórzano, J. J. Vaquero, J. M. Peña, N. Malpica, F. Del Pozo, $F$., 'Evaluation of autofocus functions in molecular cytogenetic analysis', Journal of Microscopy, 188 (3), 264-272 (1997), DOI: 10.1046/j.1365-2818.1997.2630819.x

[27] T. T. E. Yeo, S. H. Ong, Jayasooriah, R. Sinniah, 'Autofocusing for tissue microscopy', Image and Vision Computing 11/10, 629-639 (1993); doi: 10.1016/0262-8856(93)90059-P

[28] S. Nayar, Y. Nakagawa, 'Shape from focus', IEEE Transactions on Pattern Analysis and Machine Intelligence 16/8, 824-831 (1994); doi: 10.1109/34.308479

[29] H. Süße, E. Rodner, Bildverarbeitung und Objekterkennung, Springer Fachmedien Wiesbaden, Wiesbaden (2014)

[30] P. Marziliano, F. Dufaux, S. Winkler, T. Ebrahimi, 'Perceptual blur and ringing metrics: application to JPEG2000', Signal Processing: Image Communication, 19 (2), 163-172 (2004); doi:10.1016/j.image.2003.08.003

[31] E. Ong, W. Lin, Z. Lu, X. Yang, S. Yao, F. Pan, L. Jiang, F. Moschetti, 'A no-reference quality metric for measuring image blur', Proc. IEEE Symposium on Signal Processing and Its Applications, 469-472 (2003); doi: 10.1109/ISSPA.2003.1224741 